\title{
Gender Differences in Pediatric Orthopaedics: What Are the Implications for the Future Workforce?
}

\author{
Marielle A. Amoli MD, John M. Flynn MD, Eric W. Edmonds MD, \\ Michael P. Glotzbecker MD, Derek M. Kelly MD, Jeffrey R. Sawyer MD
}

Published online: 15 July 2016

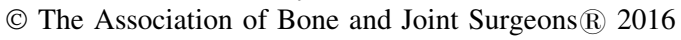

\begin{abstract}
Background Although the number of women in surgical specialties has increased dramatically over the past two decades, little research exists regarding how a surgeon's gender impacts job selection and practice models. Because the number of women specializing in pediatric orthopaedics continues to increase, it is important to understand
\end{abstract}

Each author certifies that he or she, or a member of his or her immediate family, has no funding or commercial associations (eg, consultancies, stock ownership, equity interest, patent/licensing arrangements, etc) that might pose a conflict of interest in connection with the submitted article.

All ICMJE Conflict of Interest Forms for authors and Clinical Orthopaedics and Related Research ${ }^{\mathbb{R}}$ editors and board members are on file with the publication and can be viewed on request.

Each author certifies that his or her institution approved the human protocol for this investigation and that all investigations were conducted in conformity with ethical principles of research. This work was performed at Le Bonheur Children's Hospital, Memphis, TN, USA.

M. A. Amoli, D. M. Kelly, J. R. Sawyer ( $\square)$

Department of Orthopaedic Surgery \& Biomedical Engineering,

Le Bonheur Children's Hospital, University of Tennessee-

Campbell Clinic, 1211 Union Avenue, Suite 510, Memphis,

TN 38104, USA

e-mail: jsawyer@campbellclinic.com

J. M. Flynn

Division of Orthopaedic Surgery, Children's Hospital of

Philadelphia, Philadelphia, PA, USA

E. W. Edmonds

Department of Orthopaedic Surgery, Rady Children's Hospital,

San Diego, CA, USA

M. P. Glotzbecker

Department of Orthopaedic Surgery, Boston Children's Hospital, Boston, MA, USA how one's gender affects practice choices and how this may affect the future workforce.

Questions/purposes (1) Among pediatric orthopaedic surgeons, is gender associated with choice of practice type (academic, private practice, hospital-based, solo)? (2) For men and women, what are the most common reasons for choosing a job? (3) For new graduates, do men and women have equal starting salaries? (4) Do men have a higher chance of getting job offers? (5) Is there a difference in workload (call frequency and surgical case volume) for men and women? (6) Finally, is there a difference in imminent retirement plans for men and women?

Methods The 2015 Pediatric Orthopaedic Society of North American (POSNA) Needs Assessment Survey was sent to POSNA members and a special 36-question survey was sent to recent pediatric orthopaedic fellowship graduates. Both surveys included questions about self-reported gender, practice type, reasons for job selection, and call frequency. In addition, the new graduates' survey also included questions about starting salary, job offers, and number of additional fellowships completed. Responses were analyzed by gender. Chi-square and Fisher's exact tests assessed for statistical significance.

Results Among the new graduates, women are more likely to choose academic practice (women: 13 of 18 [72\%], men: 21 of 44 [48\%], odds ratio [OR], 3.10 [confidence interval $\{\mathrm{CI}\}, 0.86-11.10], \mathrm{p}<0.001)$, whereas men are more likely to choose private practice (men: 14 of 44 [32\%o], women: one of 18 [6\%], OR, 0.12 [CI, 0.015$1.001], \mathrm{p}<0.001)$. The primary reasons for choosing a job were not different between men and women. Among the new graduates, geography/family considerations were reported as being highly important when selecting a job (men: 33 of 44 [75\%], women: 16 of 18 [89\%]) followed by academic opportunities (men: 24 of 44 [55\%], women: 
14 of 18 [78\%]). Interestingly, a higher percentage of males reported finances as being important when selecting a job (men: 23 of 44 [52\%], women: five of 18 [28\%]). For the current POSNA members, the most important reasons when choosing a job for both men and women were quality of partners (men: 168 of 408 [41\%], women: 66 of 122 [54\%]) and an interesting practice (men: 155 of 408 [38\%], women 54 of 122 [44\%]. As a result of our small sample size, there was no difference in starting salaries between men and women (< USD 350,000: men 22 of 44 [50\%], women 12 of 18 [67\%]; USD 350,000-450,000: men 11 of 44 [25\%], women six of 18 [33\%]; > USD 450,000: men eight of 44 [18\%], women zero of 18 [0\%], $p=0.131$ ). When stratified by practice type, for private practice starting salaries, over half of men (seven of 13 [54\%]) placed in the highest category of $>$ USD 400,000, whereas the single woman respondent placed in the lowest category of $<$ USD 300,000. Men were more likely to report having job offers before starting their fellowship (men: 24 of 44 [54\%], women: eight of 18 [44\%], OR, 0.67 [CI, 0.22-2.0], $\mathrm{p}=0.042$ ). Finally, among POSNA members, women reported a lower weekly surgical case volume compared with men. Of the men, 108 of 408 (26\%) reported performing more than seven surgeries per week compared with 12 of 122 women (10\%; OR, 3.4 [CI, 1.8-6.44], p < 0.001).

Conclusions Although the numbers are small given the specialized nature of pediatric orthopaedic surgery, this study has uncovered some initial gender differences regarding practice characteristics and job opportunities among pediatric orthopaedic surgeons. As more men plan to reduce their workload or retire in the next 5 years, there may be further increases in the percentage of women surgeons in the workforce, so it is important that we begin to understand what effect, if any, gender has on practice patterns, job selection, and opportunities. Also, the finding that among the new graduates more women than men are choosing careers in academic practice over private practice suggests an extraordinary opportunity to develop more female leaders and role models at major pediatric orthopaedic centers.

\section{Introduction}

During the past several decades, the number of women enrolling in medical schools has steadily increased; classes are now roughly $50 \%$ women [2]. As the number of women physicians has increased, so too has the number of women entering surgical subspecialties. Few studies, however, have looked at gender disparities within medicine, let alone within surgical subspecialties. The studies that have been done found that, despite working similar hours, women are less likely to be tenured, report lower levels of professional satisfaction, and have lower compensation than men [7, 10, 13-16]. Even fewer studies have looked at gender differences within orthopaedics. To our knowledge, this is the first study looking at gender differences within the pediatric orthopaedic workforce.

It is important to understand what effect, if any, gender has on the pediatric orthopaedic workforce, because currently $18 \%$ of active Pediatric Orthopaedic Society of North American (POSNA) members are women, which could increase to $40 \%$ by 2025 [6]. Therefore, the aim of this study was to analyze the practice characteristics, job opportunities, starting salaries, surgical caseloads, call frequency, and factors used in the job selection process to determine if gender differences exist in the current pediatric orthopaedic workforce. We also wanted to see if these gender differences exist in both recent graduates and active POSNA members who have been in practice for several years, because the increasing number of women entering pediatric orthopaedics may have a profound impact on the future pediatric orthopaedic surgery workforce.

Specific study questions addressed by this article are: (1) Among pediatric orthopaedic surgeons, is gender associated with choice of practice type (academic, private practice, hospital-based, solo)? (2) For men and women, what are the most common reasons for choosing a job? (3) For new graduates, do men and women have equal starting salaries? (4) Do men have a higher chance of getting job offers? (5) Is there a difference in workload (call frequency and surgical case volume) for men and women? (6) Finally, is there a difference in imminent retirement plans for men and women?

\section{Materials and Methods}

The 2015 electronic POSNA Needs Assessment Survey consisted of 99 questions, including self-reported gender, practice type, area of specialty, frequency and compensation for call, number of practice changes, working with residents and fellows, and mentorship opportunities available for new partners $[6,11]$. In addition, the survey also included questions regarding surgical cases per week, plans to reduce workload or retire in the next 5 years, and factors that influenced job selection. A special electronic 36question survey was sent by the POSNA leadership to the 108 recent graduates of pediatric orthopaedic fellowships (2012, 2013). Similar to the broader POSNA survey, the new graduate survey consisted of questions such as practice setting, reasons for job selection, call frequency, and number of practice changes. Unlike the POSNA survey, 
however, the new graduate survey also included questions regarding additional fellowships completed, starting salaries, and number of jobs applied for and offers received $[6,11]$.

This survey study was granted an exemption by our institutional review board.

A total of 530 POSNA members returned surveys (530 of 1228 [43\%]), of which 408 were men (77\%) and 122 were women $(23 \%)$. Of the pediatric orthopaedic new graduates' surveys, 81 of $108(52 \%)$ surveys were returned with 62 including gender demographics. Of the new graduates, 44 men $(71 \%)$ and 18 women $(29 \%)$ responded. Both surveys were analyzed by gender to determine if gender impacts the type of practice a surgeon joins, reasons for job selection, starting salaries, call frequency, workloads, and imminent plans for retirement.

When evaluating the reasons for job selection, the new graduates were asked to select the most important factors for job selection from the following: finance, geography/family, academic opportunities, frequency of call, and other. Each respondent was allowed to pick more than one factor from the mentioned list.

For the new graduates survey, starting salaries were selfreported in USD 50,000 increment ranges from < USD 200,000 to $>$ USD 600,000 . Because of the small sample size, and to better assess for any differences in starting salaries, these groups were then combined into three groups $(<$ USD 350,000 , USD $350,000-450,000$, or $>$ USD $450,000)$ and analyzed by gender as well as practice type. In addition to looking at differences in the starting salaries, the new graduate survey also looked at differences in the number and timing of job offers between men and women.

Because of differences in the survey questions regarding call frequency, the responses from the new graduate survey were grouped into no or low call (once a week or less) or frequent call (more than two nights a week). The POSNA Needs Assessment Survey responses were further divided into no call, low call (once every 7 or 8 nights), medium call (once every 5 or 6 nights), or frequent call (more than once every 4 nights).

Both surveys were then analyzed by gender, comparing women and men for the variables in question. Chi-square testing and Fisher's exact tests were used to assess for statistical significance, and $\mathrm{p}<0.05$ was determined to be statistically significant.

\section{Results}

Gender and Practice Type

Among pediatric orthopaedic surgeons entering the workforce, women are more likely to choose academic practice
Table 1. Gender differences in practice choices among new graduates

\begin{tabular}{lcc}
\hline New graduates & Men & Women \\
\hline Academic practice & $48 \%(21 / 44)$ & $72.2 \%(13 / 18)$ \\
Hospital employment & $13.6 \%(6 / 44)$ & $16.7 \%(3 / 18)$ \\
Private practice group & $31.8 \%(14 / 44)$ & $5.6 \%(1 / 18)$ \\
No answer & $4.5 \%(2 / 44)$ & $5.6 \%(1 / 18)$ \\
\hline
\end{tabular}

(women: 13 of 18 [72\%], men: 21 of 44 [48\%], odds ratio [OR], 3.10 [confidence interval $\{\mathrm{CI}\}, 0.86-11.10$ ], $\mathrm{p}<$ 0.001 ), whereas women are less likely to choose private practice (women: one of 18 [6\%], men: 14 of 44 [32\%], OR, 0.12 [CI, 0.015-1.001], $\mathrm{p}<0.001$; Table 1). With the numbers available, there was no significant difference between men and women in the percentages who chose hospital practice (women: three of 18 [17\%], men: six of 44 [14\%], OR, 1.25, p = 0.773). Gender differences regarding practice type also were noted when looking at all active POSNA members rather than just the new graduates. Among POSNA members at all stages of their career, men were more likely than women to become hospital or university employees and to choose solo practice. On the other hand, women were more likely to join already established private practice multispecialty, general orthopaedic, or pediatric orthopaedic group practices (OR, 1.83 [CI, 1.13$2.98], \mathrm{p}=0.013)$. Although the practice models chosen were significantly different, there was no significant difference in the numbers of men and women who reported working with residents (men 333 of 408 [82\%] and women 98 of 108 [80\%]) or fellows (men 178 of 408 [44\%] and women 59 of 108 [48\%]).

\section{Reasons for Job Selection}

For both men and women, regardless of where they were at in their careers, there was no difference in the primary reasons for choosing a job. Among the new graduates, geography/family considerations were reported as being highly important when selecting a job (men: 33 of 44 [75\%], women: 16 of 18 [89\%]) followed by academic opportunities (men: 24 of 44 [55\%], women: 14 of 18 [78\%]). Men, however, were more likely than women to cite finances (men: 23 of 44 [52\%], women: five of 18 [28\%]) and frequency of call (men: eight of 44 [18\%] versus women: two of 18 [11\%]) as important factors when selecting a job (Table 2). For the current POSNA members, the most important reasons when choosing a job for both men and women were quality of partners (men: 168 of 408 [41\%], women: 66 of 122 [54\%]) and an interesting practice (men: 155 of 408 [38\%], women 54 of 122 [44\%]). 
Table 2. Reasons for job selection among new graduates*

\begin{tabular}{lll}
\hline New graduates & Men & Women \\
\hline Geography/family & $75.0 \%(33 / 44)$ & $88.9 \%(16 / 18)$ \\
Academic opportunities & $54.5 \%(24 / 44)$ & $77.8 \%(14 / 18)$ \\
Finances & $52.3 \%(23 / 44)$ & $27.8 \%(5 / 18)$ \\
Frequency of call & $18.2 \%(8 / 44)$ & $11.1 \%(2 / 18)$ \\
Other & $15.9 \%(7 / 44)$ & $22.2 \%(4 / 18)$ \\
\hline
\end{tabular}

* Multiple answers allowed

Gender and Starting Salary

Among the 41 men and 18 women new graduates who responded, no differences in starting salaries were noted (< USD 350,000: men 25 of 44 [50\%], women 12 of 18 [67\%]; USD 350,000-450,000: men 11 of 44 [25\%], women six of 18 [33\%]; > USD 450,000: men eight of 44 [18\%], women zero of 18 [0\%]; $p=0.131$; Table 3). However, when stratified by practice type, for private practice starting salaries, over half of men (seven of 13 [54\%]) placed in the highest category of $>$ USD 400,000, whereas the single woman respondent placed in the lowest category of $<$ USD 300,000.

\section{Gender and Job Offer}

For the new graduates, there was no difference in the number of jobs for which men or women applied (men: 2.3 jobs, women: 2.4 jobs, $p=0.852$ ). There also was no difference in the number of offers received $(p=0.436)$ with men reporting a mean of two job offers and women reporting a mean of 1.9 offers. Despite no difference in the number of jobs applied for or the number of offers received, there was a difference noted in the timing of job offers received. Men were more likely than women to report having job offers before starting their fellowship (men: 24 of 44 [54\%], women: eight of 18 [44\%], OR, 0.617 [CI, 0.353-1.08], $\mathrm{p}=0.042$ ).

\section{Gender Workload Distribution}

When looking at workloads among all active POSNA members in regard to call volume, there were no differences for on-call workload for men and women: no call (77 of 408 [19\%] versus 15 of 122 [12\%], respectively), low to medium call (222 of 408 [55\%] versus 67 of 122 [55\%]), and frequent call (104 of 408 [26\%] versus 38 of 122 [31\%], $\mathrm{p}=0.176)$. Despite no difference in frequency of call among the active POSNA members, there was a difference between men's and women's self-reported weekly surgical caseloads with women reporting fewer surgeries per week. More specifically, 108 of 408 (26\%) men reported performing seven or more surgeries per week compared with 12 of $122(10 \%)$ women (OR, 3.4 [CI, 1.86.44], $\mathrm{p}<0.001)$.

\section{Retirement Planning by Gender}

There also was a gender difference in active POSNA members' plans to retire or reduce workload in the next 5 years. More men (men: 97 of 408 [24\%]) than women (women: 15 of 122 [12\%], OR, 0.44 [CI, 0.25-0.80], p = $0.006)$ plan to reduce their workload or retire in the next 5 years.

\section{Discussion}

Over the past several decades, more women physicians have chosen careers within surgical specialties such as orthopaedics [1,9]. According to a 2003 study by Blakemore et al. [3], the percentage of women orthopaedic residents increased from $0.6 \%$ in 1970 to nearly $9 \%$ in 2001. Of these, a large portion of women is choosing careers within pediatric orthopaedics such that $19 \%$ of the 653 active POSNA members are now women [6]. Furthermore, the number of women included in the active

Table 3. Starting salaries for new graduates

\begin{tabular}{lllll}
\hline Practice type & $<$ USD 350,000 & USD 350,000-450,000 & $>$ USD 450,000 & Overall $\mathrm{p}$ value \\
\hline Men $(\mathrm{n}=41)$ & $22(54 \%)$ & $11(27 \%)$ & $8(20 \%)$ & 0.131 \\
Academic $(\mathrm{n}=21)$ & 8 & 7 & 6 & \\
Private $(\mathrm{n}=13)$ & 4 & 2 & 7 & \\
Hospital $(\mathrm{n}=5)$ & 2 & 2 & 1 & \\
Women $(\mathrm{n}=18)$ & $12(67 \%)$ & $6(33 \%)$ & $0(0 \%)$ & \\
Academic $(\mathrm{n}=13)$ & 8 & 5 & 0 & \\
Private $(\mathrm{n}=1)$ & 1 & 0 & 0 & \\
Hospital $(\mathrm{n}=3)$ & 2 & 1 & 0 & \\
\hline
\end{tabular}


POSNA membership has been projected to reach approximately $40 \%$ by 2025 if recent membership trends continue $[6,11]$. Therefore, it is important to understand differences in career choices between men and women, including practice type and reasons for selection, compensation, and workload. Specifically, this article aimed to look at how gender affects choice of practice type, the most common reasons for choosing a job, starting salaries, job offers, call frequency, and surgical caseloads. A better understanding of how gender affects one's practice will allow professional organizations such as POSNA to closely monitor workforce and employment trends and develop strategies to promote career success and satisfaction among members.

There are several limitations to our study. First, because of the low response rates $43 \%$ for all POSNA members and $52 \%$ for the new pediatric orthopaedic fellowship graduates), which are consistent with this type of survey, our sample sizes are small. The low response rates may reflect an underlying bias, and, thus, our results may not be reflective of the group as a whole. Furthermore, the low response rates resulted in small samples sizes and low power, which limited our ability to perform statistical analysis. Another limitation was the survey design. The POSNA Needs Assessment Survey is sent annually to assess current needs of POSNA members and was not specifically designed to study gender-related differences. There were no questions specifically looking at full-time versus part-time status, clinical volume, research, family life (eg, marital status, number of children), years in practice posttraining, or salary information for the POSNA members already in practice. In addition, there were no geographic data available to determine if gender differences are uniform across the country or regionally based. Finally, all information collected was self-reported, so we were unable to check the validity of responses. Despite these limitations, our study uncovered some interesting trends regarding gender differences in practice models and career choices offering insight in the evolving pediatric orthopaedic workforce.

First, among those entering the workforce, more women are drawn to academic practice, whereas more men are drawn to private practice. The finding that women choose academic practice over private practice (in this survey, 13:1) is interesting in light of a recent study by Sharkey et al. [12] that found that, when controlled for years of membership, women are less likely to present an abstract at the annual POSNA meeting. This suggests an extraordinary opportunity to develop more women leaders and role models at major pediatric orthopaedic centers.

Second, our study found that although men and women are joining different types of practices, for the most part both genders are picking a job for similar reasons, regardless of what stage they are in their career. Among all currently active POSNA members, both genders reported that the most important reasons when choosing a job were quality of partners and an interesting practice. For the new graduates, both men and women cited family and geographic concerns as most important factors in job selection. Women cited academic opportunities as the second most important factor, whereas men prioritized finances and frequency of call. These findings may play a part in the reason women are choosing careers in academic practice, whereas more men are entering into private practice.

Because of the small sample size of new graduates as well as a lack of data on full-time versus part-time employment, we were limited in our ability to run adequate multivariate analysis on starting salaries; however, there was a trend toward women reporting lower starting salaries than men, regardless of the practice type they joined. As mentioned earlier, the different priorities placed on finances may contribute to the different starting salaries noted between men and women. Despite these limitations, our finding that women trended toward having lower starting salaries parallels a recent American Academy of Orthopaedic Surgeons survey looking at compensation and satisfaction within orthopaedics [5]. The survey found that gender disparities exist within orthopaedics in regard to salary, satisfaction of compensation, and personal life/social factors. Curry et al. [5] found that, among orthopaedic surgeons, men had higher reported salaries than women. The income disparity persisted even after accounting for age, subspecialty, hours worked, and years in practice posttraining. In addition, this study also found that a gender disparity existed in regard to feelings of equal compensation with $70 \%$ of men and only $38 \%$ of women reporting that both genders were equally compensated. These findings also parallel findings from previous research that showed gender disparities in terms of salary and professional satisfaction in plastic surgeons, colorectal surgeons, pediatric surgeons, otolaryngologists, hospitalists, and primary care providers [4, 8, 10, 17].

Although no difference was noted between the number of jobs for which men and women applied or the number of job offers received, our study did find that men received job offers earlier than women with the majority of the men having an offer before fellowship. Again, it is hard to fully understand these findings because we have no information regarding the timing of job application for men and women. Perhaps men started the job search process earlier in their training than women, which would explain why more men had offers before their fellowships started compared with women. Also, there was no geographic information obtained to see if the differences noted were regional or national.

Although the larger POSNA survey found that women surgeons reported fewer surgical cases per week, it is hard 
to understand how this translates to the overall workloads. Again, there were no questions in the survey regarding nonsurgical workloads such as clinical volumes or time in research. In addition, there was no information regarding the types of surgical cases performed. More complex surgical cases require more time, and thus a surgeon may have fewer self-reported surgical cases a week despite an equal overall workload. In addition, despite differences noted in self-reported surgical volumes among all active POSNA members, there was no difference noted in on-call frequency, suggesting that there may be no difference in overall workloads between the genders. Furthermore, the surveys did not ask about full-time versus part-time employment, making it difficult to fully understand the differences in self-reported surgical volumes.

Finally, there was a gender difference noted in active POSNA members' plans to retire or reduce workload in the next 5 years. The finding that more men plan to reduce their workload or retire in the next 5 years may further increase the percentage of women surgeons in the workforce. Therefore, it is important that we begin to develop better understandings of the gender differences that exist and how these differences impact the workforce.

In conclusion, there are differences between men and women when it comes to career choices, practice models, and priorities in pediatric orthopaedics. By better understanding these differences, we can not only help to develop more women leaders and role models at major pediatric orthopaedic centers, but also improve professional and personal satisfaction. This study serves as a benchmark for the future studies looking at gender, geography, employment status (full- versus part-time), years in practice posttraining, marital status, and number of children, which are warranted to better understand these complex issues.

Acknowledgments We thank Kay Daugherty for her editorial support.

\section{References}

1. American Academy of Orthopaedic Surgeons. Orthopaedic practice in the U.S. 2014. Available at http://www.aaos.org/ downloadasset.aspx?id=31147. Accessed July 7, 2016.

2. Association of American Medical Colleges. Diversity in medical education: facts and figures, 2008. Available at: https://members.
aamc.org/eweb/upload/Diversity\%20in\%20Medical\%20Education Facts\%20and\%20Figures\%202012.pdf. Accessed November 19, 2015.

3. Blakemore LC, Hall JM, Biermann JS. Women in surgical residency training programs. J Bone Joint Surg Am. 2003;85:24772480.

4. Caniano DA, Sonnino RE, Paolo AM. Keys to career satisfaction: insights from a survey of women pediatric surgeons. J Pediatr Surg. 2004;39:984-990.

5. Curry EJ, Rieger R, Matzkin EG. Compensation and satisfaction in orthopaedics. Orthopaedic Journal at Harvard Medical School. June 2015. Available at: http://www.orthojournalhms. org/currentIssue.html. Accessed November 19, 2015.

6. Glotzbecker MP, Shore BJ, Fletcher ND, Larson AN, Hydorn CR, Sawyer JR; Practice Management Committee of the Pediatric Orthopaedic Society of North America. Early career experience of pediatric orthopaedic fellows: what to expect and need for their services. J Pediatr Orthop. 2016;36:429-432.

7. Grandis JR, Gooding WE, Zamboni BA, Wagener MM, Drenning SD, Miller L, Doyle KJ, Mackinnon SE, Wagner RL. The gender gap in a surgical subspecialty analysis of career and lifestyle factors. Arch Otolaryngol Head Neck Surg. 2004;130:695-702.

8. Halperin TJ, Werler MM, Mulliken JB. Gender differences in the professional and private lives of plastic surgeons. Ann Plast Surg. 2010;64:775-779.

9. Lewis VO, Scher SA, O'Connor MI. Women in orthopaedicsway behind the number curve. J Bone Joint Surg Am. 2012;94:e30(1-7).

10. Sanfey H, Fromson J, Mellinger J, Rakinic J, Williams M, Williams B. Surgeons in difficulty: an exploration of differences in assistance-seeking behaviors between male and female surgeons. J Am Coll Surg. 2015;221:621-627.

11. Sawyer JR, Jones KC, Copley LA, Chambers S; POSNA Practice Management Committee. Pediatric orthopaedic workforce in 2014: current workforce and projections for the future. J Pediatr Orthop. 2015 Oct 30 [Epub ahead of print].

12. Sharkey MS, Feinn RS, Tatte VV, Carter CW, Cassese TT. Disproportionate participation of males and females in academic pediatric orthopaedics: an analysis of abstract authorship at POSNA 2009-2013. J Pediatr Orthop. 2016;36:433-436.

13. Waljee JF, Chang KW, Kim HM, Gyetko MR, Quint EH, Lukacs NW, Woolliscroft JO, Chung KC. Gender disparities in academic practice. Plast Reconstr Surg. 2015;136:380e-387e.

14. Wallace AE, Weeks WB. Differences in income between male and female primary care physicians. J Am Med Womens Assoc. 2002;57:180-184.

15. Weaver AC, Wetterneck TB, Whelan CT, Hinami K. A matter of priorities? Exploring the persistent gender pay gap in hospital medicine. J Hosp Med. 2015;10:486-490.

16. Weeks WB Wallace AE. Differences in the annual incomes of emergency physicians related to gender. Acad Emerg Med. 2007; 14:434-440.

17. Zutshi M, Hammel J, Hull T. Colorectal surgeons: gender differences in perceptions of a career. $J$ Gastrointest Surg. 2010;14:830-843. 